\title{
Performance of albumin-bilirubin score in prediction of hepatic encephalopathy in cirrhotic patients with acute variceal bleeding
}

Rehab Elsayed Elsafty(D, Abdallah Ahmed Elsawy* (D), Ahmed Fawzy Selim(i) and Atef Mohamed Taha(D)

\begin{abstract}
Background: Hepatic encephalopathy exacerbates the morbidity, delays hospital discharge, and increases the rate of readmissions of cirrhotic patients, particularly those are admitted by acute variceal bleeding. We evaluated the performance of albumin-bilirubin score in prediction of hepatic encephalopathy in cirrhotic patients with acute variceal bleeding, in comparison to Child-Pugh and MELD scores. This prospective cohort study was conducted on 250 cirrhotic patients who were consecutively presented by acute variceal bleeding in the period from January to December 2020 at Tanta university emergency hospital. Albumin-bilirubin, Child-Pugh, and MELD scores were measured at admission, and then all patients were followed up for 4 weeks after endoscopic bleeding control for possible occurrence of hepatic encephalopathy
\end{abstract}

Results: Albumin-bilirubin, Child-Pugh, and MELD scores had significant performances in prediction of hepatic encephalopathy in cirrhotic patients with acute variceal bleeding; in this regard, albumin-bilirubin score had the highest accuracy (AUC 0.858, Cl 0.802-0.914, sig 0.000) followed by Child-Pugh score (AUC 0.654, Cl 0.574-0.735, sig 0.001 ) and then MELD score (AUC 0.602, Cl 0.519-0.686, sig 0.031). The cumulative incidence of hepatic encephalopathy in cirrhotic patients with albumin-bilirubin grade 3 was found to be significantly more than that present in albumin-bilirubin grade 2; most of these hepatic encephalopathy cases occurred in the first 2 weeks of follow-up period.

Conclusions: Albumin-bilirubin score has a significant performance in risk prediction of hepatic encephalopathy in cirrhotic patients with acute variceal bleeding better than Child-Pugh and MELD scores. Albumin-bilirubin grades could be used as a risk stratifying tool to triage cirrhotic patients who will benefit from early discharge after bleeding control and those patients who will benefit from prophylactic measures for hepatic encephalopathy.

Keywords: Albumin-bilirubin score, Prediction, Hepatic encephalopathy, Variceal bleeding, Cirrhosis

\section{Background}

Hepatic encephalopathy (HE) exacerbates the morbidity and delays hospital discharge as well as increases the rate of hospital readmissions of cirrhotic patients, particularly those are admitted by acute variceal bleeding (AVB) [1-5]. The varying severity of liver

\footnotetext{
* Correspondence: abdallahelsawy@hotmail.com

Department of Internal Medicine, Faculty of Medicine, Tanta University, Tanta, Egypt
}

\section{Springer Open}

decompensation is related to both the incidence and prevalence of hepatic encephalopathy in cirrhotic patients $[1,6]$; the underlying residual liver functions are commonly and widely evaluated by Child-Pugh score [7]; however, this score has some limitations due to the interrelation between albumin and ascites and the subjective assessment of encephalopathy and ascites [8]. The new albumin-bilirubin (ALBI) score was developed previously by Johnson et al.; this 
model depends on two objectively evaluated parameters (serum albumin and serum bilirubin); it assesses the degree of underlying hepatic reserve function in cirrhotic patients and has a high degree of performance in prediction of mortality due to liver failure [9]. This score was widely validated in many reports [10-13]. In this study, we assessed the performance of albuminbilirubin score in prediction of hepatic encephalopathy in cirrhotic patients with acute variceal bleeding, in comparison to Child-Pugh and MELD scores.

\section{Methods}

\section{Study design}

This is a prospective cohort study that was conducted in accordance to strengthening the reporting of observational studies in epidemiology (STROBE) guidelines [14].

\section{Source of data and potential eligible population}

This study was conducted at adult gastroenterology unit of internal medicine department at Tanta university hospitals, in the period from January 2020 to December 2020 on a total of 319 patients who were admitted consecutively by acute upper gastro-intestinal bleeding (hematemesis and/or melena); all patients were resuscitated and then they were evaluated by upper gastrointestinal (GIT) endoscopy for assessment and control of bleeding source. Our eligible participants were recruited in accordance to our inclusion and exclusion criteria that were assessed within the first $24 \mathrm{~h}$ after admission.

\section{Exclusion and inclusion criteria}

Fifty-four patients were excluded as they did not fulfill study inclusion criteria (31 patients were excluded due to non-variceal upper GIT bleeding, 9 patients excluded as they were clinically unfit for upper GIT endoscopy, 10 patients were excluded due to failure of endoscopic control of variceal bleeding, 4 patients were excluded because cirrhosis was not confirmed), five patients were excluded as they were presented by hepatic encephalopathy before upper GIT endoscopy, three patients were excluded as they died shortly after admission during preoperative resuscitation, and seven patients were excluded as they refused follow-up in our unit. Therefore, the eligibility criteria were fulfilled in only 250 cirrhotic patients who were presented consecutively by acute variceal bleeding and were controlled successfully by upper GIT endoscopy.

\section{Data collection}

Each patient was meticulously evaluated after admission and resuscitation with or without blood transfusion, and then upper GIT endoscopy was done within $12 \mathrm{~h}$ from the occurrence of hematemesis and/or melena for fit patients to diagnose and control of bleeding source; all the operators were highly experienced physicians at our endoscopy unit. The following first admission criteria were evaluated for all patients who fulfilled our inclusion criteria: demographic criteria (age and sex), clinical criteria (presence of hematemesis and/or melena, ascites, spleen diameter, variceal grading, endoscopic modality of variceal bleeding control and their need for blood transfusion), and laboratory criteria (AST, ALT, s.albumin. s.bilirubin, INR, s.creatinine, platelet count, hemoglobin). The collected patients' laboratory data were used to calculate Child-Pugh, MELD, and albumin-bilirubin (ALBI) scores. Albumin-bilirubin score was calculated using the following formula $[(\log 10$ bilirubin in $\mu \mathrm{mol} / \mathrm{L} \times 0.66)+$ (albumin in $\mathrm{g} / \mathrm{L}$ $x-0.085)]$. Levels $\leq-2.6$ was considered as ALBI grade 1, levels $>-1.39$ was considered as ALBI grade 3 , and other levels were considered as ALBI grade 2 [9]. All patients were followed up for 4 weeks for possible occurrence of hepatic encephalopathy; we considered the day of hematemesis or the day of first appearance of melena as the initiation time point of follow-up period; all patients were followed up firstly at our emergency unit for few days and then completed the follow-up period at internal wards of our gastroenterology unit or at our outpatient clinic through scheduled examinations every week, as illustrated in study participants' flow diagram (Fig. 1). All patients received broad spectrum antibiotics routinely for at least 1 week after endoscopy as a prophylactic measure for post-endoscopic bacterial infection.

\section{Statistical analysis methods}

We used IBM SPSS, version 23 statistic software (IBM, NY, USA), for both summarization and statistical analysis of our collected data. The mean and standard deviation (SD) was used for normally distributed quantitative data while the median and interquartile range (IQR) was calculated for abnormally distributed quantitative data; however, all qualitative data were tabulated as frequency and relative frequency tables. Receiver operating characteristic (ROC) curves were calculated for our ALBI, Child-Pugh, and MELD scores, and their areas under the ROC curve (AUC) were computed. The cumulative incidence functions for hepatic encephalopathy in-between ALBI grades were illustrated using Kaplan-Meier curve and analyzed using the Log Rank test. $P$ values less than 0.05 were considered statistically significant.

\section{Results}

Table 1 of our results shows the main demographic, clinical, and laboratory baseline criteria for study eligible 
Potential eligible population:

319 patients were consecutively presented to our emergency gastroenterology unit by upper GIT bleeding (hematemesis and/or melena)

\section{Exclusion criteria: $(n=69)$}

- Unfulfillment of study inclusion criteria $(n=54)$

- Presentation with hepatic encephalopathy $(n=5)$.

- Death during resuscitation before endoscopy $(n=3)$

- Refuse of follow up in our unit $(n=7)$

250 cirrhotic patients who were consecutively presented by variceal bleeding that wellcontrolled by upper GIT endoscopy.

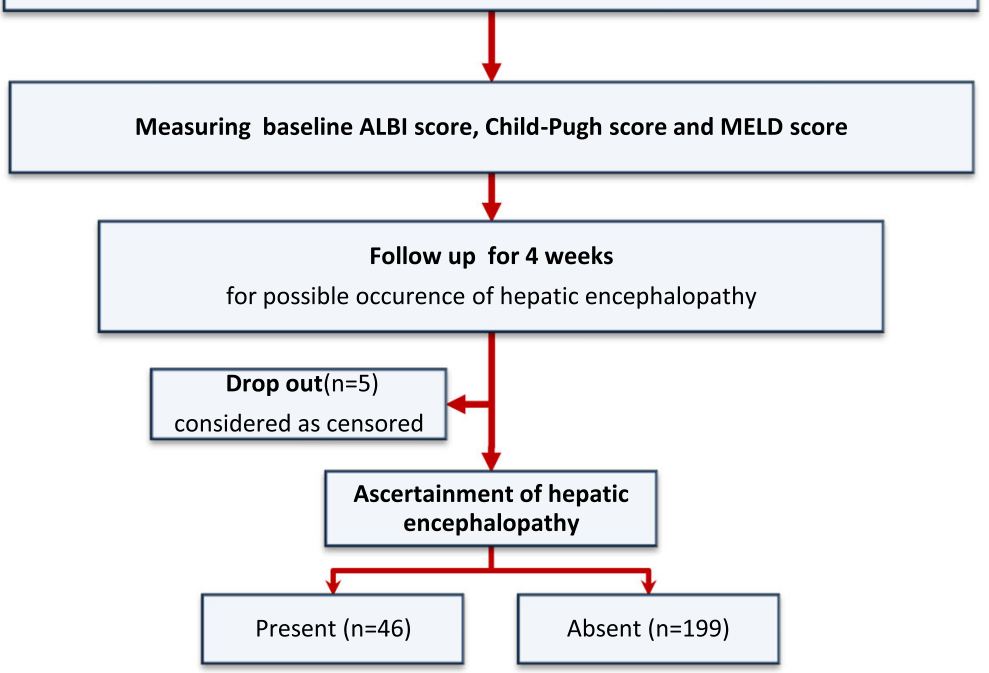

Fig. 1 Participants' flow diagram

population as well as their baseline scores of liver functions (Child-Pugh, MELD, and ALBI scores).

Table 2 of our results shows cross-tabulation of hepatic encephalopathy versus deferent grades of ALBI score along the follow-up period; the cumulative incidence of HE in our study was found to be $18.3 \%$ (46 HE cases) after 4 weeks follow-up time; 39 of these HE cases (84.8\%) occurred in patients with ALBI grade 3 while the remaining $7 \mathrm{HE}$ cases (15.2\%) occurred in patients with ALBI grade 2.

Figure 2 of our results illustrates the receiver operating criteria curve for baseline ALBI, Child-Pugh, and MELD scores in prediction of hepatic encephalopathy with AUC and their respective confidence intervals and significance values (AUC 0.858, 0.654, and 0.602; CI 0.802-0.914, $0.574-0.735$, and $0.519-0.686$ with sig $0.000,0.001$, and 0.031 for ALBI, Child-Pugh, and MELD scores respectively.
Figure 3 of our results illustrates cumulative incidence function for different ALBI grades as a risk factor predicting hepatic encephalopathy, in which there was a significant difference between ALBI grade 2 and ALBI grade $3(\mathrm{sig}=0.000)$.

\section{Discussion}

Acute variceal bleeding is a known predisposing risk factor for hepatic encephalopathy in cirrhotic patients $[1,3]$; this may exacerbate the morbidity and delay hospital discharge as well as increase the rate of hospital readmissions and mortality in those patients [1-6]; consequently, early administration of appropriate prophylactic measures for $\mathrm{HE}$ in risky patients with AVB may mitigate both morbidity and mortality burden as well as alleviate the possible shortage of hospital places especially in emergency situations. 
Table 1 Main baseline participants' criteria:

\begin{tabular}{|c|c|c|c|c|}
\hline \multicolumn{2}{|c|}{ Baseline criteria for eligible population } & \multicolumn{3}{|l|}{ Value } \\
\hline \multicolumn{5}{|l|}{ Demographic criteria } \\
\hline - Age (years) & & Mean (SD) & 58 & $(9.1)$ \\
\hline \multirow[t]{3}{*}{ - Sex } & - Male & Count (\%) & 160 & $(64 \%)$ \\
\hline & - Female & Count (\%) & 90 & $(36 \%)$ \\
\hline & - Total & Count (\%) & 250 & $(100 \%)$ \\
\hline \multicolumn{5}{|l|}{ Patients clinical criteria } \\
\hline \multirow[t]{3}{*}{ - Presentation } & - Hematemesis & Count (\%) & 52 & $21 \%$ \\
\hline & - Melena & Count (\%) & 37 & $15 \%$ \\
\hline & - Combined hematemesis and melena & Count (\%) & 161 & $64 \%$ \\
\hline \multicolumn{2}{|l|}{ - Previous variceal bleeding } & Count (\%) & 105 & $42 \%$ \\
\hline \multirow[t]{3}{*}{ - Variceal grading } & - Grade 1 & Count (\%) & 10 & $4 \%$ \\
\hline & - Grade 2 & Count (\%) & 102 & $41 \%$ \\
\hline & - Grade 3 & Count (\%) & 138 & $55 \%$ \\
\hline \multirow[t]{2}{*}{ - Variceal bleeding control } & - EBL & Count (\%) & 42 & $17 \%$ \\
\hline & • EIT & Count (\%) & 208 & $83 \%$ \\
\hline \multicolumn{2}{|l|}{ - Need for blood transfusion } & Count (\%) & 163 & $65 \%$ \\
\hline \multirow[t]{3}{*}{ - Ascites } & - No ascites & Count (\%) & 33 & $(13.2 \%)$ \\
\hline & - Mild to moderate & Count (\%) & 141 & $(56.4 \%)$ \\
\hline & - Massive & Count (\%) & 76 & $(30.4 \%)$ \\
\hline \multicolumn{2}{|l|}{ - Spleen diameter $(\mathrm{cm})$} & Median (IQR) & 15 & (3) \\
\hline \multicolumn{2}{|l|}{$\cdot \mathrm{HCC}$} & Count (\%) & 22 & $(9 \%)$ \\
\hline \multirow[t]{4}{*}{ - Etiology of cirrhosis } & $\cdot \mathrm{CHC}$ & Count (\%) & 196 & $(78.4 \%)$ \\
\hline & $\cdot \mathrm{CHB}$ & Count (\%) & 21 & $(8.4 \%)$ \\
\hline & - $\mathrm{CHC}$ and $\mathrm{CHB}$ & Count (\%) & 9 & $(3.6 \%)$ \\
\hline & - Other & Count (\%) & 24 & $(9.6 \%)$ \\
\hline \multicolumn{5}{|l|}{ Laboratory parameters } \\
\hline \multicolumn{2}{|l|}{ • ALT (IU/L) } & Mean (SD) & 43.3 & $(11.66)$ \\
\hline \multicolumn{2}{|l|}{ • AST $(I U / L)$} & Mean (SD) & 47.6 & $(10.73)$ \\
\hline \multicolumn{2}{|l|}{ - S. albumin (gm/l) } & Mean (SD) & 28.9 & $(3.42)$ \\
\hline \multicolumn{2}{|l|}{ - S. bilirubin (mg/dl) } & Median (IQR) & 2.5 & $(1.4)$ \\
\hline \multicolumn{2}{|l|}{ - INR } & Median (IQR) & 2.1 & $(0.30)$ \\
\hline \multicolumn{2}{|l|}{ - S. creatinine (mg/dl) } & Median (IQR) & 1.2 & $(0.2)$ \\
\hline \multicolumn{2}{|l|}{ - Platelets $\left(\times 10^{3} / \mathrm{mm}^{3}\right)$} & Median (IQR) & 145 & (54) \\
\hline \multicolumn{2}{|l|}{ - Hemoglobin (g/dl) } & Median (IQR) & 9.6 & $(0.9)$ \\
\hline \multicolumn{5}{|c|}{ Evaluated scores for liver functions } \\
\hline \multicolumn{2}{|l|}{ - Child-Pugh score } & Median (IQR) & 10 & (3) \\
\hline \multicolumn{2}{|l|}{ - MELD score } & Median (IQR) & 19.8 & $(4.4)$ \\
\hline - ALBI score & & Median (IQR) & -1.4 & $(0.6)$ \\
\hline - ALBI grade & - ALBI grade 1 & Count (\%) & 0 & $(0 \%)$ \\
\hline & - ALBI grade 2 & Count (\%) & 118 & $(47.2 \%)$ \\
\hline & - ALBI grade 3 & Count (\%) & 132 & $(52.8 \%)$ \\
\hline
\end{tabular}

EBL endoscopic band ligation, EIT endoscopic injection therapy, HCC hepatocellular carcinoma, CHC chronic hepatitis C, CHB chronic hepatitis B, ALT alanine aminotransferase, AST aspartate aminotransferase, INR international normalized ratio, MELD modified end-Istage liver disease, $A L B I$ albumin-bilirubin, SD standard deviation, IQR interquartile range 
Table 2 ALBI grades versus hepatic encephalopathy cross-tabulation

\begin{tabular}{|c|c|c|c|c|c|c|c|c|c|}
\hline & & \multicolumn{6}{|c|}{ Hepatic encephalopathy } & \multirow[t]{3}{*}{ Sig. } & \multirow{3}{*}{$\begin{array}{l}\text { Risk estimate } \\
(95 \% \mathrm{Cl})\end{array}$} \\
\hline & & \multicolumn{5}{|c|}{ Present $(n=46)$} & \multirow{2}{*}{$\begin{array}{l}\text { Absent ( } n \\
=204)\end{array}$} & & \\
\hline & & 1st week & 2nd week & 3rd week & 4th week & Total & & & \\
\hline ALBI grade $\mathbf{2}(n=118)$ & Count (\%) & $2(28.6 \%)$ & $4(57.1 \%)$ & $1(14.3 \%)$ & $0(0.0 \%)$ & $7(5.9 \%)$ & $111(94.1 \%)$ & $<0.0005$ & $4.98(2.31-10.75)$ \\
\hline ALBI grade $\mathbf{3}(n=132)$ & Count (\%) & $14(35.9 \%)$ & $18(46.2 \%)$ & $5(12.8 \%)$ & $2(5.1 \%)$ & 39 (29.5\%) & $93(70.5 \%)$ & & \\
\hline
\end{tabular}

ALBI grade albumin-bilirubin grade

In this respect, the risk stratification of those cirrhotic patients with AVB at hospital admissions could be helpful for early prediction of possible occurrence of $\mathrm{HE}$ even after bleeding control; these predictors were evaluated in many reports $[15,16]$. One of these risk stratifying factors is the underlying liver function which is commonly assessed by Child-Pugh score [7]; however, this score may be limited by the interrelation between albumin and ascites and the subjective assessment of encephalopathy and ascites [8]. In our study, we used the new ALBI score for the underlying liver functions as a risk stratifying factor for prediction of $\mathrm{HE}$ in cirrhotic patients with AVB; we hypothesized that this score has a better performance in comparison to Child-Pugh and MELD scores as it depends on only two objectively assessed parameters (serum albumin and serum bilirubin).

First of all, we found that the cumulative incidence of HE in our patients were about $18.3 \%$ within 4 weeks follow-up period, and the reported cumulative incidences of HE after AVB have great variability; it was reported as $16.9 \%$ by Win J et al. [17], 31.4\% by Fouad TR

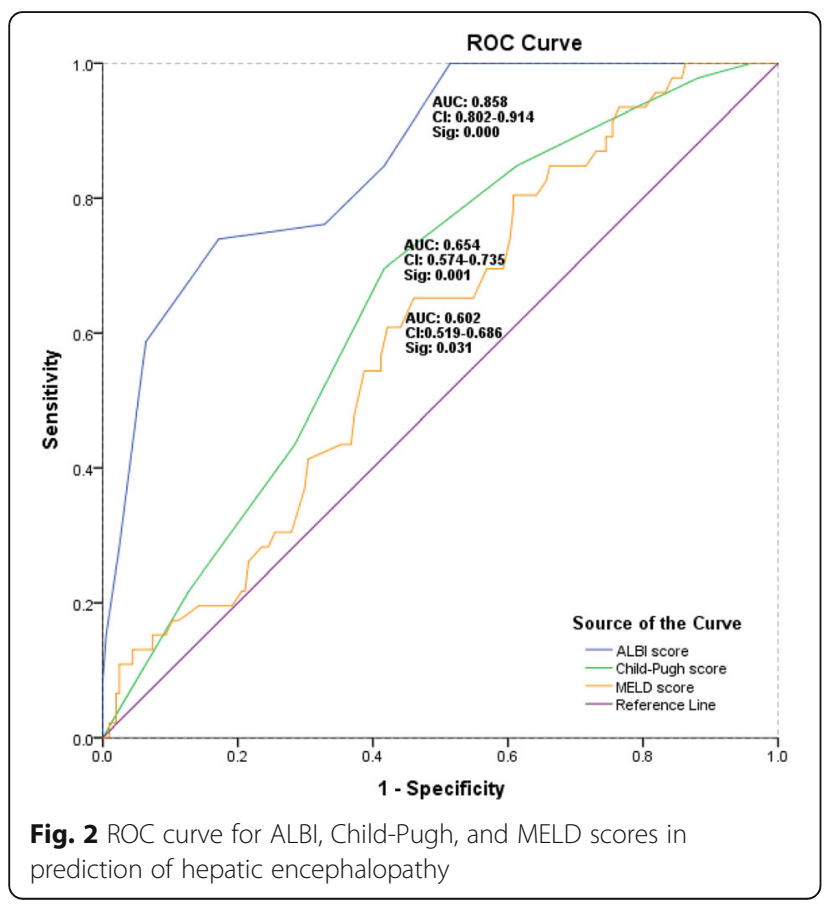

et al. [18], $40 \%$ by Sharma $\mathrm{P}$ et al. [19], and $54.5 \%$ by Higuera-de-la-Tijera F et al. [20]; this wide discrepancy may be due to the wide variability of the underlying reserve liver functions, variability in follow-up periods, and variability in modalities of variceal bleeding control. Various prophylactic measures of HE in cirrhotic patients after acute upper GIT bleeding were studied in many reports and were found to significantly improve the incidence rates of $\mathrm{HE}$ in those patients; these measures aim to eliminate the blood from GIT to decrease the absorption of its toxic products which is the main mechanism of HE after AVB [17-20].

As regards the accuracy of ALBI score in prediction of HE in cirrhotic patients with AVB in comparison to both Child-Pugh and MELD scores, we found that all of these scores had a significant performance; however, we found that ALBI score had the highest performance followed by Child-Pugh score then MELD score. Fouad TR et al. [18] reported a similar conclusion to ours as they identified the possible risk prediction of HE for ALBI score in comparable to Child-Pugh and MELD scores.

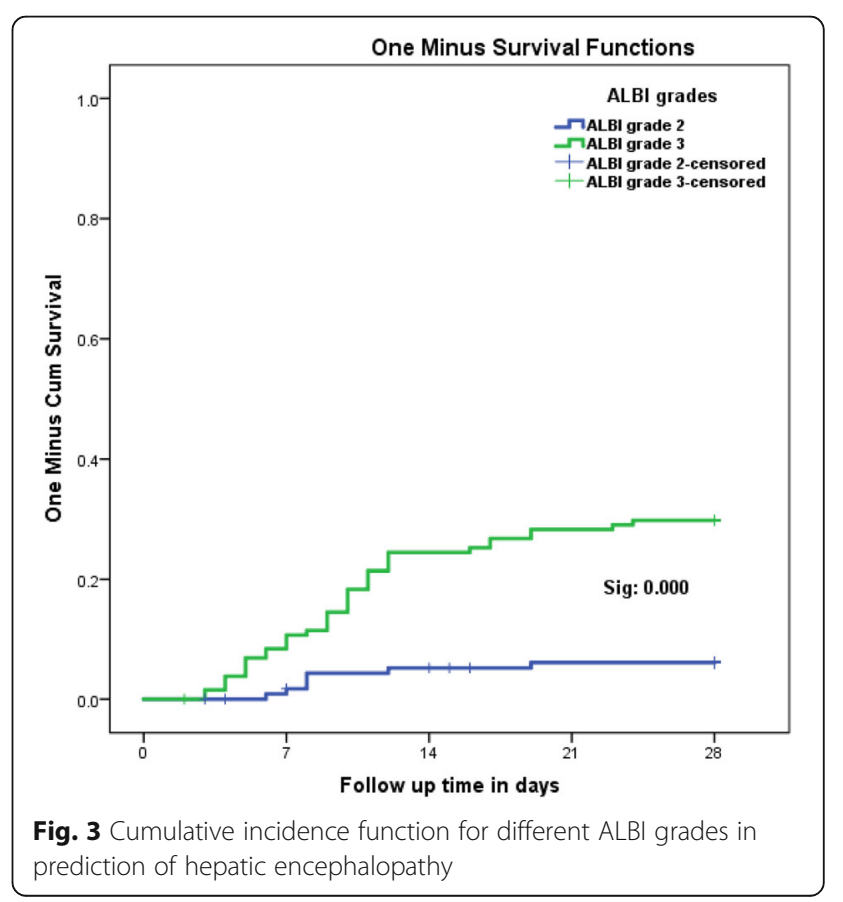


In our study, we used ALBI grades for risk stratifying of our patients, and we found that the cumulative incidence function of HE in cirrhotic patients with ALBI grade 3 was significantly more than that present in ALBI grade 2; most of these HE cases occurred in the first 2 weeks of follow-up period, so we could suggest that early hospital discharge for those cirrhotic patients after good control of AVB could be possible in absence of ALBI grade 3; this is the same concept that was reported by Fouad TR et al. [18]. At the same time, we could encourage the administration of prophylactic measures for those cirrhotic patients with ALBI grade 3 who are admitted with AVB.

Our study has some limitations; the first limitation is the uni-centericity of this study, and the second is that the methods of follow-up were not the same for all patients; however, we tried to select the most appropriate method for each patient; the third limitation is that we did not assess the possible hazard of rebleeding; however, we found only 11 cases $(4.4 \%)$ of mild rebleeding episodes that were appropriately controlled. Lastly, we did not correlate between ALBI score and different stages of HE; however, we focused in our study on the possible predictive performance of ALBI score for possible occurrence of $\mathrm{HE}$.

\section{Conclusion}

We could conclude that albumin-bilirubin score has a significant performance in risk prediction of hepatic encephalopathy in cirrhotic patients with acute variceal bleeding better than both Child-Pugh and MELD scores. Albumin-bilirubin grades could be used as a risk stratifying tool to triage cirrhotic patients who will benefit from early discharge after bleeding control and those patients who will benefit from prophylactic measures for hepatic encephalopathy.

\section{Abbreviations \\ ALBI: Albumin-bilirubin; ALT: Alanine aminotransferase; AST: Aspartate aminotransferase; AUC: Area under the curve; AVB: Acute variceal bleeding; CHB: Chronic hepatitis B; CHC: Chronic hepatitis C; Cl: Confidence interval; EBL: Endoscopic band ligation; EIT: Endoscopic injection therapy; GIT: Gastrointestinal; HCC: Hepatocellular carcinoma; HE: Hepatic encephalopathy; INR: International normalized ratio; IQR: Interquartile range; MELD: Modified end-stage liver disease; ROC: Receiver operating characteristics; SD: Standard deviation; Sig: Significance}

\section{Acknowledgements}

We would like to thank all staff members in Gastroenterology Unit of Internal Medicine department at Tanta University Hospital that assisted us in this study.

\section{Authors' contributions}

All authors read and approved the final manuscript, according to the following respective roles of each author: REE shared in the study conception and design, data collection, and data interpretation. AAE shared in the study conception and design, data collection, data analysis, and data interpretation and is the corresponding author. AFS shared in the study conception and design, data collection, and data interpretation. AMT shared in the study conception and design, data collection, and data interpretation.
Funding

Not applicable

\section{Availability of data and materials}

The datasets used and/or analyzed during the current study are available from the corresponding author on reasonable request.

\section{Declarations}

\section{Ethics approval and consent to participate}

The present study was conducted in accordance with the Declaration of Helsinki. The study protocol was approved by the ethics committee of Tanta Faculty of Medicine (No: 19/12/33594). All patients provided written informed consent. The results of the research were used only in scientific purposes and not in any other aims.

Consent for publication

Not applicable

Competing interests

The authors declare that they have no competing interests.

Received: 28 January 2021 Accepted: 4 March 2021

Published online: 12 March 2021

\section{References}

1. American Association for the Study of Liver Diseases; European Association for the Study of the Liver (2014) Hepatic encephalopathy in chronic liver disease: 2014 practice guideline by the European Association for the Study of the Liver and the American Association for the Study of Liver Diseases. J Hepatol 61(3):642-659

2. Bleibel W, Al-Osaimi AM (2012) Hepatic encephalopathy. Saudi J Gastroenterol 18(5):301-309. https://doi.org/10.4103/1319-3767.101123

3. Patidar KR, Bajaj JS (2015) Covert and overt hepatic encephalopathy: diagnosis and management. Clin Gastroenterol Hepatol 13(12):2048-2061. https://doi.org/10.1016/j.cgh.2015.06.039

4. Volk ML, Tocco RS, Bazick J, Rakoski MO, Lok AS (2012) Hospital readmissions among patients with decompensated cirrhosis. Am J Gastroenterol 107(2):247-252. https://doi.org/10.1038/ajg.2011.314

5. Seraj SM, Campbell EJ, Argyropoulos SK, Wegermann K, Chung RT, Richter JM (2017) Hospital readmissions in decompensated cirrhotics: factors pointing toward a prevention strategy. World J Gastroenterol 23(37):68686876. https://doi.org/10.3748/wjg.v23.i37.6868

6. Del Piccolo F, Sacerdoti D, Amodio P, Bombonato G, Bolognesi M, Mapelli D et al (2003) Central nervous system alterations in liver cirrhosis: the role of portal-systemic shunt and portal hypoperfusion. Metab Brain Dis 18(1):5162. https://doi.org/10.1023/A:1021930702815

7. Pugh R, Murray-lyon I, Dawson J (1973) Transection of the oesophagus for bleeding oesophageal varices. Br J Surg 60(8):646-649. https://doi.org/10.1 002/bjs.1800600817

8. Wang YY, Zhao XH, Ma L, Ye JZ, Wu FX, Tang J, You XM, Xiang BD, Li LQ (2018) Comparison of the ability of Child-Pugh score, MELD score, and ICGR15 to assess preoperative hepatic functional reserve in patients with hepatocellular carcinoma. J Surg Oncol 118(3):440-445. https://doi.org/10.1 002/jso.25184

9. Johnson PJ, Berhane S, Kagebayashi C, Satomura S, Teng M, Reeves HL, O'Beirne J, Fox R, Skowronska A, Palmer D, Yeo W, Mo F, Lai P, Iñarrairaegui M, Chan SL, Sangro B, Miksad R, Tada T, Kumada T, Toyoda H (2015) Assessment of liver function in patients with hepatocellular carcinoma: a new evidence-based approach-the ALBI grade. J Clin Oncol 33(6):550-558. https://doi.org/10.1200/JCO.2014.57.9151

10. Hiraoka A, Kumada T, Michitaka K, Kudo M (2019) Newly proposed ALBI grade and ALBI-T score as tools for assessment of hepatic function and prognosis in hepatocellular carcinoma patients. Liver Cancer 8(5):312-325. https://doi.org/10.1159/000494844

11. Wang YY, Zhong JH, Su ZY, Huang JF, Lu SD, Xiang BD, Ma L, Qi LN, Ou BN, Li LQ (2016) Albumin-bilirubin versus Child-Pugh score as a predictor of outcome after liver resection for hepatocellular carcinoma. Br J Surg 103(6): 725-734. https://doi.org/10.1002/bjs.10095

12. Toyoda H, Lai PB, O'Beirne J, Chong CC, Berhane S, Reeves H et al (2016) Long-term impact of liver function on curative therapy for hepatocellular 
carcinoma: application of the ALBI grade. Br J Cancer 114(7):744-750. https://doi.org/10.1038/bjc.2016.33

13. Hiraoka A, Kumada T, Nouso K, Tsuji K, Itobayashi E, Hirooka M, Kariyama K, Ishikawa T, Tada T, Toyoda H, Kawasaki H, Hiasa Y, Michitaka K (2016) Proposed new sub-grouping for intermediate-stage hepatocellular carcinoma using albumin-bilirubin grade. Oncology. 91(3):153-161. https:// doi.org/10.1159/000447061

14. Vandenbroucke JP, von Elm E, Altman DG, Gøtzsche PC, Mulrow CD, Pocock SJ, Poole C, Schlesselman JJ, Egger M, STROBE Initiative (2014) Strengthening the Reporting of Observational Studies in Epidemiology (STROBE): explanation and elaboration. Int J Surg 12(12):1500-1524. https:// doi.org/10.1016/j.jjsu.2014.07.014

15. Rattanasupar A, Tiawijit N, Rachatapantanakorn B (2014) Predictive factor for hepatic encephalopathy in cirrhotic patients who presented with acute variceal bleeding. J Med Assoc Thail 97(6):567-573

16. Kumar AS, Sibia RS (2015) Predictors of in-hospital mortality among patients presenting with variceal gastrointestinal bleeding. Saudi I Gastroenterol 21(1):43-46. https://doi.org/10.4103/1319-3767.151226

17. Wen J, Liu Q, Song J, Tong M, Peng L, Liang H (2013) Lactulose is highly potential in prophylaxis of hepatic encephalopathy in patients with cirrhosis and upper gastrointestinal bleeding: results of a controlled randomized trial. Digestion. 87(2):132-138. https://doi.org/10.1159/000346083

18. Fouad TR, Abdelsameea E, Abdel-Razek W, Attia A, Mohamed A, Metwally K, Naguib M, Waked I (2019) Upper gastrointestinal bleeding in Egyptian patients with cirrhosis: post-therapeutic outcome and prognostic indicators. J Gastroenterol Hepatol 34(9):1604-1610. https://doi.org/10.1111/jgh.14659

19. Sharma P, Agrawal A, Sharma BC, Sarin SK (2011) Prophylaxis of hepatic encephalopathy in acute variceal bleed: a randomized controlled trial of lactulose versus no lactulose. J Gastroenterol Hepatol 26(6):996-1003. https://doi.org/10.1111/j.1440-1746.2010.06596.x

20. Higuera-de-la-Tijera F, Servín-Caamaño Al, Salas-Gordillo F, Pérez-Hernández $\mathrm{JL}$, Abdo-Francis JM, Camacho-Aguilera J et al (2018) Primary prophylaxis to prevent the development of hepatic encephalopathy in cirrhotic patients with acute variceal bleeding. Can J Gastroenterol Hepatol 2018:3015891

\section{Publisher's Note}

Springer Nature remains neutral with regard to jurisdictional claims in published maps and institutional affiliations.

\section{Submit your manuscript to a SpringerOpen ${ }^{\circ}$ journal and benefit from:}

- Convenient online submission

- Rigorous peer review

- Open access: articles freely available online

- High visibility within the field

- Retaining the copyright to your article

Submit your next manuscript at $\boldsymbol{\nabla}$ springeropen.com 